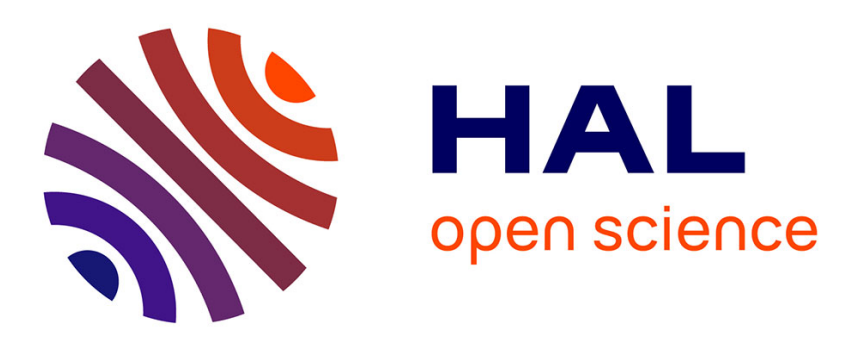

\title{
On three-dimensional ALE finite element model for simulating deformed interstitial medium in the presence of a moving needle
}

\author{
Yannick Deleuze, Marc Thiriet, Tony W.H. Sheu
}

\section{- To cite this version:}

Yannick Deleuze, Marc Thiriet, Tony W.H. Sheu. On three-dimensional ALE finite element model for simulating deformed interstitial medium in the presence of a moving needle. Computers and Fluids, 2016, 10.1016/j.compfluid.2016.08.001 . hal-01355051

\section{HAL Id: hal-01355051 \\ https://hal.sorbonne-universite.fr/hal-01355051}

Submitted on 22 Aug 2016

HAL is a multi-disciplinary open access archive for the deposit and dissemination of scientific research documents, whether they are published or not. The documents may come from teaching and research institutions in France or abroad, or from public or private research centers.
L'archive ouverte pluridisciplinaire HAL, est destinée au dépôt et à la diffusion de documents scientifiques de niveau recherche, publiés ou non, émanant des établissements d'enseignement et de recherche français ou étrangers, des laboratoires publics ou privés. 


\title{
On Three-Dimensional ALE Finite Element Model For Simulating Deformed Interstitial Medium in the Presence of a Moving Needle
}

\author{
Yannick Deleuze $^{\mathrm{a}, \mathrm{b}, *}$, Marc Thiriet ${ }^{\mathrm{a}, \mathrm{c}, \mathrm{d}}$, Tony W.H. Sheu ${ }^{\mathrm{b}, \mathrm{e}}$ \\ ${ }^{a}$ Sorbonne Universités, UPMC Univ Paris 06, UMR 7598, Laboratoire Jacques-Louis \\ Lions, F-75005, Paris, France. \\ ${ }^{b}$ Department of Engineering Science and Ocean Engineering, National Taiwan \\ University, No. 1, Sec. 4, Roosevelt Road, Taipei, Taiwan. \\ ${ }^{c}$ CNRS, UMR 7598, Laboratoire Jacques-Louis Lions, F-75005, Paris, France. \\ ${ }^{d}$ INRIA-Paris-Rocquencourt, EPC REO, Domaine de Voluceau, BP105, 78153 Le \\ Chesnay Cedex. \\ ${ }^{e}$ Center for Advanced Study in Theoretical Sciences, National Taiwan University, No. 1, \\ Sec. 4, Roosevelt Road, Taipei, Taiwan.
}

\begin{abstract}
The effects of inserted needle on the subcutaneous interstitial flow is studied.

Our goal is to describe the physical stress affecting cells during acupuncture needling. The convective Brinkman equations are considered to describe the flow through a fibrous medium. Three-dimensional simulations are carried out by employing an ALE finite element model. Numerical studies illustrate the acute physical stress developed by the implantation of a needle.
\end{abstract}

Keywords: Finite element method, ALE, FreeFem++, acupuncture, Brinkman model, interstitial fluid flow

\footnotetext{
*Corresponding author

Email addresses: yannick.deleuze@ljll.math.upmc.fr (Yannick Deleuze), marc.thiriet@upmc.fr (Marc Thiriet), twhsheu@ntu.edu.tw (Tony W.H. Sheu)
} 


\section{Introduction}

In recent years, computational techniques have been widely used by researchers to investigate and simulate biological flow within three dimensional context. Applications include blood flow models, air flow models in the respi-

5 ratory tract, interstitial flow models, and chemical mediators transport. Most of the structure and fluid interactions have been considered with simplified rigid wall or deformable wall models.

Methods to predict flows that account for moving domains or domain deformability using the finite element method are based on fixed mesh methods or moving mesh methods. On the one hand, fixed mesh methods include the immersed boundary formulation [1] which relies on the description of solid phase by adding a force vector to the governing equations. A similar approach, known as the fictitious domain formulation [2, 3], is based on the use of Lagrange multipliers to enforce kinematic condition on the solid phase or alternatively based on a penalty method [3]. Both methods track solid phase with a characteristic function or a level set function. These methods are well adapted to moving bodies in the fluid or fluid-structure computation with interface of a highly geometric complexity. The latest method has been implemented with FreeFem ++ [4]. On the other hand, moving mesh methods include the Lagrangian method, the moving finite element (MFE) method [5, 6], the deformation map method [7], the Geometric Conservation Law (GCL) method [8], the space/time method [9-12], and the Arbitrarily Lagrangian-Eulerian (ALE) method [13 15] for the solution of fluid dynamic problems. Note that the space-time finite element method can also be implemented in FreeFem ++ in $1 \mathrm{D}$ and $2 \mathrm{D}$. 
Significant progress has been made in recent years in solving fluid-structure interaction problems in deformable domains using the ALE method. The mathematically rigorous ALE framework has been well accepted to be applicable to simulate transport phenomena in time and allows some freedom in the description of mesh motion. A theoretical analysis of the ALE method can be found in [16, 17]. However, ALE equations are computationally expensive when considering a large domain because of the necessity of continuously updating the geometry of the fluid and structural mesh. Interface tracking with time discretization also raises some implementation questions.

35 The implementation of the ALE method can be done in FreeFem ++ [18.

Study of biological flows plays a central role in acupuncture research. For a description of the underlying acupuncture mechanism, one can refer to [1921]. Interstitial flow models take into account interstitial fluid, cell membrane interaction, and fiber interactions [22]. Mastocytes, among other cells, are able to respond to fluidic stimuli via mechanotransduction pathways leading to the degranulation and liberation of chemical mediators [23]. Degranulation mechanisms include interaction of the cell membrane with interstitial and cytosolic flow [24. Ion transport in narrow ion channels is another challenging task to model. Indeed, degranulation of chemical mediators upon stimulation can be triggered by a rapid $\mathrm{Ca}^{2+}$ entry in the cytosol [25].

Modeling the three-dimensional interstitial flow in tissues is extremely challenging for a large number of reasons: a complex geometry of the tissue, an accurate constitutive description of the behavior of the tissue, and flow rheology are only few examples. Macroscopic models developed for incorposo rating complex microscopic structure are essential for applications [22, 25- 
28]. In the context of acupuncture, the interstitial flow has been modeled by the Brinkman equations in two-dimensional fixed domain [27, 28] and two-dimensional deformable domain [19].

In this paper, a porous medium formulation of the interstitial fluid is presented for modeling mastocyte-needle interaction in deformable connective tissues. This formulation is based on the conventional ALE characteristic/Galerkin finite element model for an unsteady flow thought a porous medium modeled by the incompressible Brinkman's equations in a threedimensional moving domain. The motion of the needle in the fluid is taken into account. The main features of the model can be summarized as follows:

1. The loose connective tissue of the hypodermis is constituted of scattered cells immersed in extracellular matrix. The extracellular matrix contains relatively sparse fibers and abundant interstitial fluid. The interstitial fluid contains water, ions and other small molecules. Such a fluid corresponds to plasma without macromolecules and interacts with the ground substance, thereby forming a viscous hydrated gel that can stabilize fiber network [29, 30].

2. The Darcy law is used to approximate fibers of the media as a continuum and allows us to compute the actual microscopic flow phenomena that occur in the fibrous media.

3. Brinkman's law then allows us to describe the flow field around solid bodies such as the embedded cells in extracellular matrix.

4. Transient convective Brinkman's equations [31 33] are applied to simulate interstitial flow in a fibrous medium driven by a moving needle 75 [19]. 
Although the previously stated approach cannot give information on microscopic events, it can describe macroscale flow patterns in porous media. Focus is given to the effects of interstitial fluid flow during implantation of an acupuncture needle until the tip has reached the desired location within the hypodermis. The objective of this work is to give a description of the physical stress (shear stress and pressure) influencing tissue and cells.

\section{Methods}

On a microscopic scale, the interstitial tissues are composed of fluid, cells, and solid fibers. The interstitial fluid contains water, ions and other small molecules. Such a fluid corresponds to plasma without macromolecules [22]. It interacts with the ground substance to form a gel-like medium.

A model taking into account individual fibers and cell adhesion complexes is already a falsification of the reality. Moreover, it is very costly from the computational viewpoint. When considering an organized homogeneous matrix of fibers, computation of such a model shows the microscopic fluctuations of the fluid shear stress at the protein level [34].

Due to biological complexity, the interstitium is considered as a fluid-filled porous material [22]. The interstitial flow is simulated using the incompressible convective Brinkman equation. The phenomenological model cannot give information on unneeded microscopic events but the Darcy equation can describe macroscale flow patterns in porous media.

\subsection{Flow equations}

The governing equations of the unsteady flow of an incompressible fluid through a porous medium (with mass density $\rho$, dynamic viscosity $\mu$, and 
100

$$
\begin{aligned}
\frac{\rho}{\alpha_{f}}\left(\frac{\partial \overline{\mathbf{u}}}{\partial t}+\overline{\mathbf{u}} \cdot \nabla\left(\frac{\overline{\mathbf{u}}}{\alpha_{f}}\right)\right)-\mu \nabla^{2} \overline{\mathbf{u}}+\frac{1}{\alpha_{f}} \nabla\left(\alpha_{f} p_{f}\right) & =-\frac{\mu}{P} \overline{\mathbf{u}} \quad \text { in } \Omega(t),(1) \\
\nabla \cdot \overline{\mathbf{u}} & =0 \\
\overline{\mathbf{u}}(\mathbf{x}, 0) & =\overline{\mathbf{u}}_{0}(\mathbf{x})
\end{aligned}
$$

where $-\frac{\mu}{P} \overline{\mathbf{u}}$ denotes the Darcy drag, $P$ the Darcy permeability, $\overline{\mathbf{u}}$ the averaged velocity vector, and $p_{f}$ the pressure. The averaged velocity is defined as

$$
\overline{\mathbf{u}}=\alpha_{f} \mathbf{u}_{f}
$$

where $\mathbf{u}_{f}$ is the fluid velocity and

$$
\alpha_{f}=\frac{\text { fluid volume }}{\text { total volume }}
$$

is the fluid volume fraction. This volume fraction corresponds to the effec105 tive porosity of the medium. The fluid fractional volume $\alpha_{f}$ is taken as a space-dependent parameter to model the distinguished properties around an acupoint.

The system of equations (1,2) is applied to the case of a flow driven by the motion of a needle in the deformable domain $\Omega(t)$ [19]. The domain boundary 110 can be decomposed into four surfaces: the needle boundary denoted by $\Gamma_{1}$, an impervious boundary (wall) denoted by $\Gamma_{2}$, the mastocyte membrane denoted by $\Gamma_{3}$, and the open boundary on the sides denoted by $\Gamma_{4}$. The classical noslip condition is applied to the needle surface $\Gamma_{1}$, the rigid wall $\Gamma_{2}$, and the cell surface $\Gamma_{3}$. At the outer boundary $\Gamma_{4}$ a traction-free boundary condition 
is prescribed. Thus, the entire set of boundary conditions reads as

$$
\begin{aligned}
\overline{\mathbf{u}} & =\mathbf{v}_{\text {needle }}, \text { on } \Gamma_{1}, \\
\overline{\mathbf{u}} & =0, \text { on } \Gamma_{2}, \\
\overline{\mathbf{u}} & =0, \text { on } \Gamma_{3}, \\
-\mu \nabla \overline{\mathbf{u}} \cdot \mathbf{n}+p_{f} \mathbf{n} & =0, \text { on } \Gamma_{4} .
\end{aligned}
$$

\subsection{Finite element model}

The governing equations in section 2.2.1 are solved using the finite element software FreeFem $++[35]$. This code programs the discrete equations derived from the finite element weak formulation of the problem presented in section $120 \quad 2.2 .3$ using a characteristic/Galerkin model to stabilize convection terms.

\subsubsection{Scaling and setting for numerical simulations}

$\mathrm{L}$ denotes the characteristic length that is the needle width and $\mathrm{V}$ is the characteristic velocity set to be the needle maximum velocity. Rescaling the variables leads to

$$
\mathbf{x}^{\prime}=\frac{\mathbf{x}}{\mathrm{L}}, \quad t^{\prime}=\frac{t}{(\mathrm{~L} / \mathrm{V})}, \quad p^{\prime}=\frac{p_{f}}{\left(\rho V^{2}\right)}, \quad \mathbf{u}^{\prime}=\frac{\overline{\mathbf{u}}}{\mathrm{V}}
$$

In the resulting dimensionless form, after removing the prime in the rescaled variables, the dimensionless incompressible convective Brinkman equations read as

$$
\begin{aligned}
\frac{1}{\alpha_{f}} \frac{\partial \mathbf{u}}{\partial t}+\frac{1}{\alpha_{f}} \mathbf{u} \cdot \boldsymbol{\nabla}\left(\frac{\mathbf{u}}{\alpha_{f}}\right)-\frac{1}{\operatorname{Re}} \nabla^{2} \mathbf{u}+\frac{1}{\alpha_{f}} \nabla\left(\alpha_{f} p\right) & =-\frac{1}{\operatorname{DaRe}} \mathbf{u} \\
\boldsymbol{\nabla} \cdot \mathbf{u} & =0 .
\end{aligned}
$$


where Re is the Reynolds number and Da is the Darcy number. The previous dimensionless parameters are defined as

$$
\mathrm{Re}=\frac{\rho \mathrm{LV}}{\mu}, \quad \mathrm{Da}=\frac{P}{\mathrm{~L}^{2}} .
$$

In considering the above dimensionless governing equations, the normalized boundary conditions on the domain boundary are prescribed as

$$
\begin{aligned}
\mathbf{u} & =\mathbf{v} & & \text { on } \Gamma_{1}, \\
\mathbf{u} & =0 & & \text { on } \Gamma_{2}, \\
\mathbf{u} & =0 & & \text { on } \Gamma_{3}, \\
-\frac{1}{\operatorname{Re}} \boldsymbol{\nabla} \mathbf{u} \cdot \mathbf{n}+p \mathbf{n} & =0 & & \text { on } \Gamma_{4} .
\end{aligned}
$$

\subsubsection{ALE implementation on moving meshes}

In the present paper, the ALE framework built in FreeFem++ is employed to compute the flow in the moving domain. In the current problem setting, domain is thus exactly known at each time so that an area preserving mesh can be precisely generated.

The framework of the ALE approach employed is briefly described below. Let $\Omega(t)$ be the domain at each time $t$ with regular boundary $\partial \Omega(t)$. In the Eulerian description, the fluid is described by

$$
\mathbf{u}(\mathbf{x}, t) \text { and } p(\mathbf{x}, t), \forall \mathbf{x} \in \Omega(t) \text {. }
$$

To follow a moving domain, one can define the ALE map as

$$
\tilde{\mathcal{A}}: \tilde{\omega} \times \mathbb{R}^{+} \rightarrow \mathbb{R}^{2} \quad(\tilde{\mathbf{x}}, t) \rightarrow \tilde{\mathcal{A}}(\tilde{\mathbf{x}}, t):=\tilde{\mathcal{A}}_{t}
$$


such that $\omega(t)=\tilde{\mathcal{A}}(\tilde{\omega}, t)$, where $\tilde{\omega}$ is the reference computational domain. Given an ALE field $\tilde{q}: \tilde{\omega} \times \mathbb{R}^{+} \rightarrow \mathbb{R}$, its Eulerian description is given by

$$
\forall \mathbf{x} \in \Omega(t), q(\mathbf{x}, t)=\tilde{q}\left(\tilde{\mathcal{A}}_{t}^{-1}(\mathbf{x}), t\right)
$$

In ALE framework, the computational domain velocity (or ALE velocity or grid velocity) is defined as

$$
\tilde{\mathbf{a}}(\tilde{\mathbf{x}}, t)=\frac{\partial \tilde{\mathcal{A}}}{\partial t}(\tilde{\mathbf{x}}, t), \quad \forall \tilde{\mathbf{x}} \in \tilde{\omega}
$$

so that we can get

$$
\mathbf{a}(\mathbf{x}, t)=\tilde{\mathbf{a}}\left(\tilde{\mathcal{A}}_{t}^{-1}, t\right) .
$$

The ALE time-derivative is defined as

$$
\left.\frac{\partial q}{\partial t}\right|_{\tilde{\mathcal{A}}}=\frac{\mathrm{d}}{\mathrm{d} t} q(\tilde{\mathcal{A}}(\tilde{\mathbf{x}}, t), t),
$$

and the following identity holds

$$
\left.\frac{\partial q}{\partial t}\right|_{\tilde{\mathcal{A}}}=(\mathbf{a} \cdot \boldsymbol{\nabla}) q+\frac{\partial q}{\partial t} .
$$

A general method is used to construct the mapping or, equivalently, the domain velocity a. The domain velocity is computed by solving the following Laplace equation subjected to the Dirichlet boundary condition [36]

$$
-\nabla^{2} \mathbf{a}=0, \quad \mathbf{a}_{\left.\right|_{\partial \Omega}}=\mathbf{v} .
$$

In the ALE framework, the equations 11,12 , subject to a prescribed needle motion, become

$$
\begin{aligned}
\left.\frac{\partial\left(\mathbf{u} / \alpha_{f}\right)}{\partial t}\right|_{\tilde{\mathcal{A}}}+\left(\left(\frac{\mathbf{u}}{\alpha_{f}}-\mathbf{a}\right) \cdot \boldsymbol{\nabla}\right) \frac{\mathbf{u}}{\alpha_{f}}-\frac{1}{\operatorname{Re}} \nabla^{2} \mathbf{u}+\frac{1}{\alpha_{f}} \nabla\left(\alpha_{f} p\right)=-\frac{\mathbf{u}}{\operatorname{DaRe}}, \\
\boldsymbol{\nabla} \cdot \mathbf{u}=0 .
\end{aligned}
$$

The solutions $\mathbf{u}$ and $p$ are sought subject to the initial condition (3) and the boundary conditions (7, described in section 2.2.1. 


\subsubsection{Finite element discretization}

The convective incompressible Brinkman equations are approximated with the method of characteristics for the nonlinear convection term and a Galerkin method for the rest of the spatial derivative terms. The time discretization gives

$$
\begin{aligned}
\frac{1}{\Delta t}\left(\frac{\mathbf{u}^{n+1}}{\alpha_{f}}-\left(\frac{\mathbf{u}^{n}}{\alpha_{f}}\right) \circ \mathbf{X}^{n}\right)-\frac{1}{\operatorname{Re}} \nabla^{2} \mathbf{u}^{n+1}+\frac{1}{\alpha_{f}} \nabla\left(\alpha_{f} p^{n+1}\right) & =-\frac{\mathbf{u}^{n+1}(28)}{\text { DaRe }} \operatorname{Re}^{(29)} \\
\nabla \cdot \mathbf{u}^{n+1}+\varepsilon p^{n+1} & =0,
\end{aligned}
$$

in $\Omega^{n+1}$. Note that $\mathbf{X}^{n}$ is approximated by $\mathbf{X}^{n} \approx \mathbf{x}-\left(\frac{\mathbf{u}^{n}}{\alpha_{f}}-\mathbf{a}^{n}\right)(\mathbf{x}) \Delta t$. Note that a small stabilization parameter epsilon is introduced following the so-called artificial compressibility method introduced in 37] and [38.

For all $\varphi \in H^{1 / 2}\left(\Gamma_{1}\right)$, let us introduce the product space

$$
V_{\varphi}=\left\{(\mathbf{w}, q) \in\left[H^{1}(\Omega)\right]^{2} \times L^{2}(\Omega), \mathbf{w}=\varphi \text { on } \Gamma_{1}, \mathbf{w}=0 \text { on } \Gamma_{2}\right\}
$$

Let

$$
(a, b)=\int_{\Omega^{n+1}} a b \mathrm{~d} \mathbf{x}
$$

The weak formulation becomes the following finite dimensional linear system: find $\left(\mathbf{u}^{n+1}, p^{n+1}\right) \in V_{g}$ such that

$$
\begin{array}{r}
\frac{1}{\Delta t}\left(\frac{\mathbf{u}^{n+1}}{\alpha_{f}}-\left(\frac{\mathbf{u}^{n}}{\alpha_{f}}\right) \circ \mathbf{X}^{n}, \mathbf{w}\right)+\frac{1}{\operatorname{Re}}\left(\frac{1}{\alpha_{f}} \nabla \mathbf{u}^{n+1}, \boldsymbol{\nabla} \mathbf{w}\right) \\
-\left(\alpha_{f} p^{n+1}, \boldsymbol{\nabla} \cdot\left(\frac{\mathbf{w}}{\alpha_{f}}\right)\right)+\frac{1}{\operatorname{DaRe}}\left(\mathbf{u}^{n+1}, \mathbf{w}\right)=0, \\
\left(\boldsymbol{\nabla} \cdot \mathbf{u}^{n+1}, q\right)+\varepsilon\left(p^{n+1}, q\right)=0,
\end{array}
$$

for all $(\mathbf{w}, q) \in V_{0}$. 
The Taylor-Hood $\mathbb{P}_{2}-\mathbb{P}_{1}$ elements are adopted to ensure satisfaction of the LBB stability condition [39]. Note that temporal accuracy order of the presented characteristic/Galerkin method is one. Meshes are generated within FreeFem ++ and mesh adaptation is performed prior to simulations so as to improve mesh quality around the needle and the cell.

\subsection{Validation}

Let the interaction of an oscillating circular cylinder with a fluid at rest be considered. The problem is to find the velocity vector field $\mathbf{u}$ and the pressure $p$ of a flow satisfying the incompressible Navier-Stokes equations in the domain $\Omega=[-l, l] \times[-h, h]$ with no-slip boundary conditions on the cylinder and traction-free boundary condition on the border of the physical domain.

The horizontal velocity of the cylinder of diameter $D$ is given by $u_{c}(t)=$ ${ }_{160}-U \cos (2 \pi f t)$, where $U=2 \pi A f$. At each time step, the mesh is moved according to the displacement $\mathbf{a} \Delta t$, where $\mathbf{a}$ is the solution of 25. As soon as the mesh is moved, the computed $\mathbf{a}^{n}$ and $\mathbf{u}^{n}$, that are defined in the previous mesh, are then pushed to the new mesh without interpolation following the scheme proposed in [18].

165 Numerical simulation of flow is carried out for $\operatorname{Re}=100$ and $K C:=$ $\frac{U}{D f}=5$, where $U=1, D=1, T:=1 / f=5$, and $A=5 / 2 \pi$. In figures 1 and 2, good comparison between the computed solution and experimental data from [40] is shown at two different times that correspond to the phases $7 \pi / 6$ and $11 \pi / 6$. 

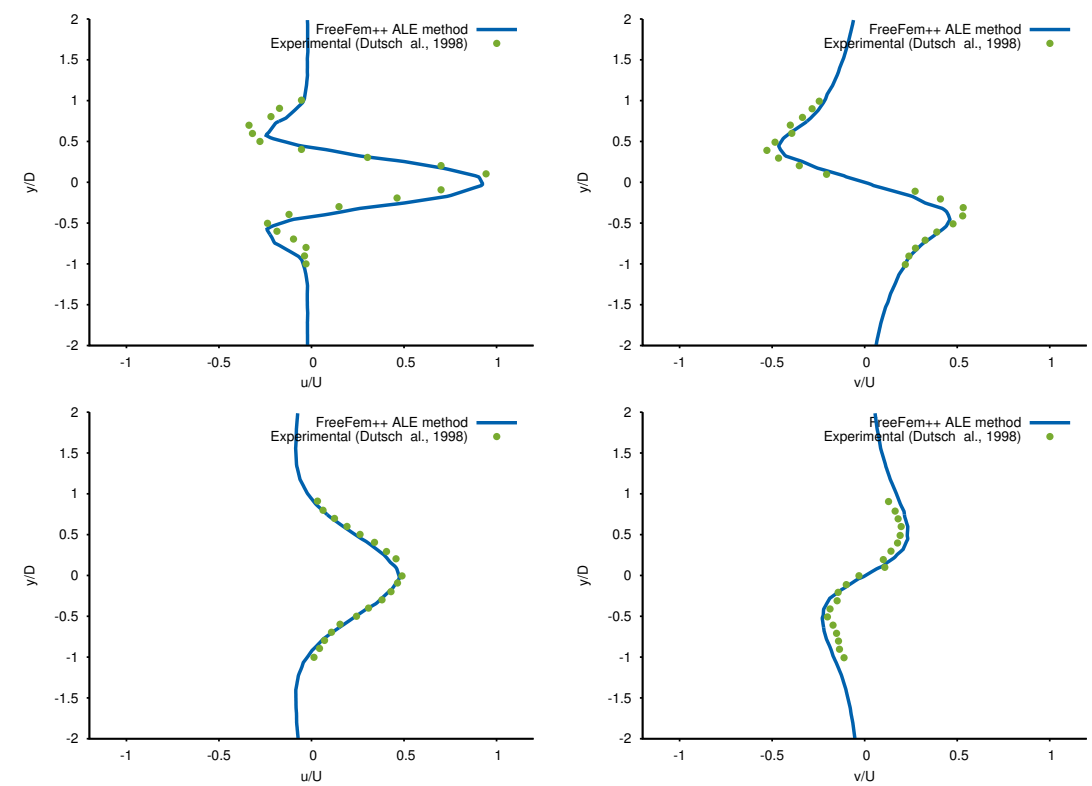

Figure 1: Comparison of the computed and referenced solutions along the line $x=-0.6 * D$ (top) and $x=1.2 * D$ (bottom) for the values of $\mathbf{u}=(u, v)$ at $t=\frac{55}{12} T$.

\section{Results}

In the present work, the needling direction is perpendicular to the skin surface. In practice, it is possible that the needling direction is oblique to the skin surface. The simulation results show that the insertion of an acupuncture needle can influence interstitial fluid flow. The computed velocity field shows that at a location away from the needle, the effect of the stress field on the meshwork vanishes (Fig. 3). Furthermore, when the needle reaches its maximum speed, the interstitial pressure gradient becomes higher at a location close to the needle tip (Fig. 4). The changes in the interstitial fluid flow and the high pressure gradient can affect the activities of the mastocyte pools in the stimulated area. Local mastocyte pools can be activated 

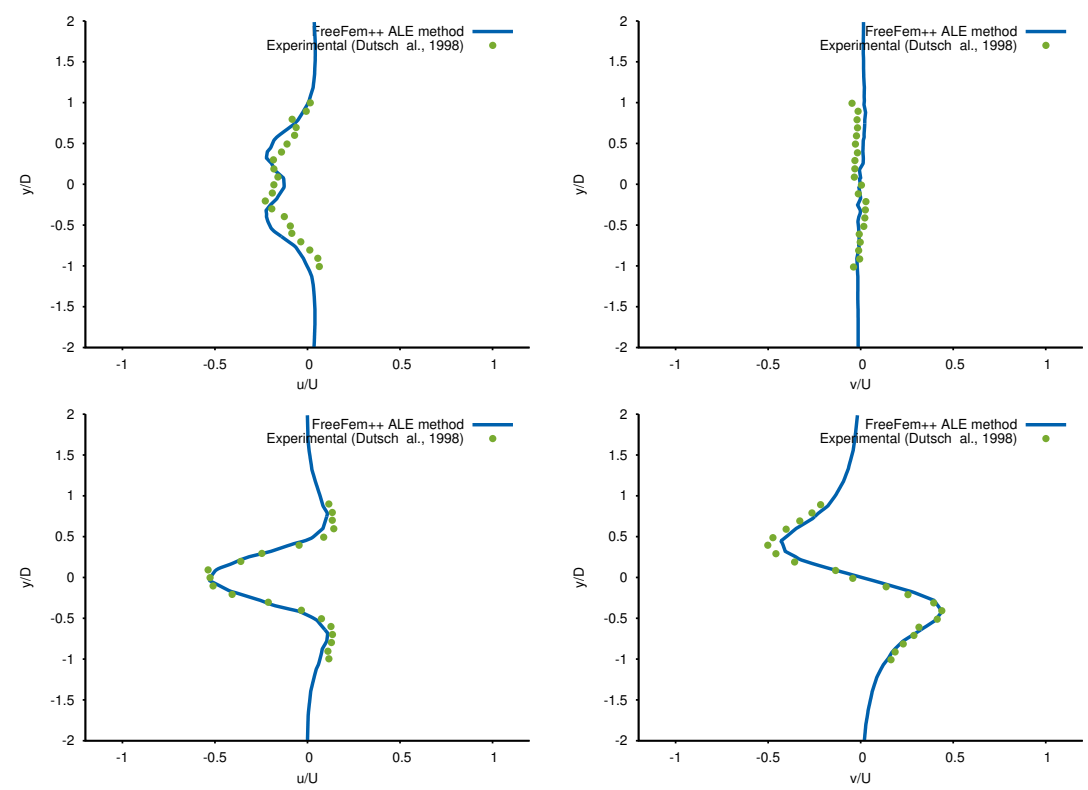

Figure 2: Comparison of the computed and referenced solutions along the line $x=-0.6 * D$ (top) and $x=1.2 * D$ (bottom) for the values of $\mathbf{u}=(u, v)$ at $t=\frac{59}{12} T$.

in regions close to the needle and remain granulated outside this region of triggered mechanical stress.

Another subject of interest is the effects of the fluidic stimuli on an interstitial cell. Local mechanical forces can trigger the activation of mechanoresponsive proteins on the cell surface [20, 41] so that $\mathrm{Ca}^{++}$is allowed to enter the cytosol via pressure and shear stress gated ion channels. Simulations are carried out by considering fixed cells and no-slip boundary condition prescribed at the cell surface. Figure 5 shows the pressure contours on the surface of a cell added closely to the needle. Figure 6 shows the streamlines and the shear stress along the cell surface. The pressure and the shear stress on the cell surface appear to be higher in the region closest to the needle tip. Fluctuation of the pressure and the time varying shear stress suggest that 

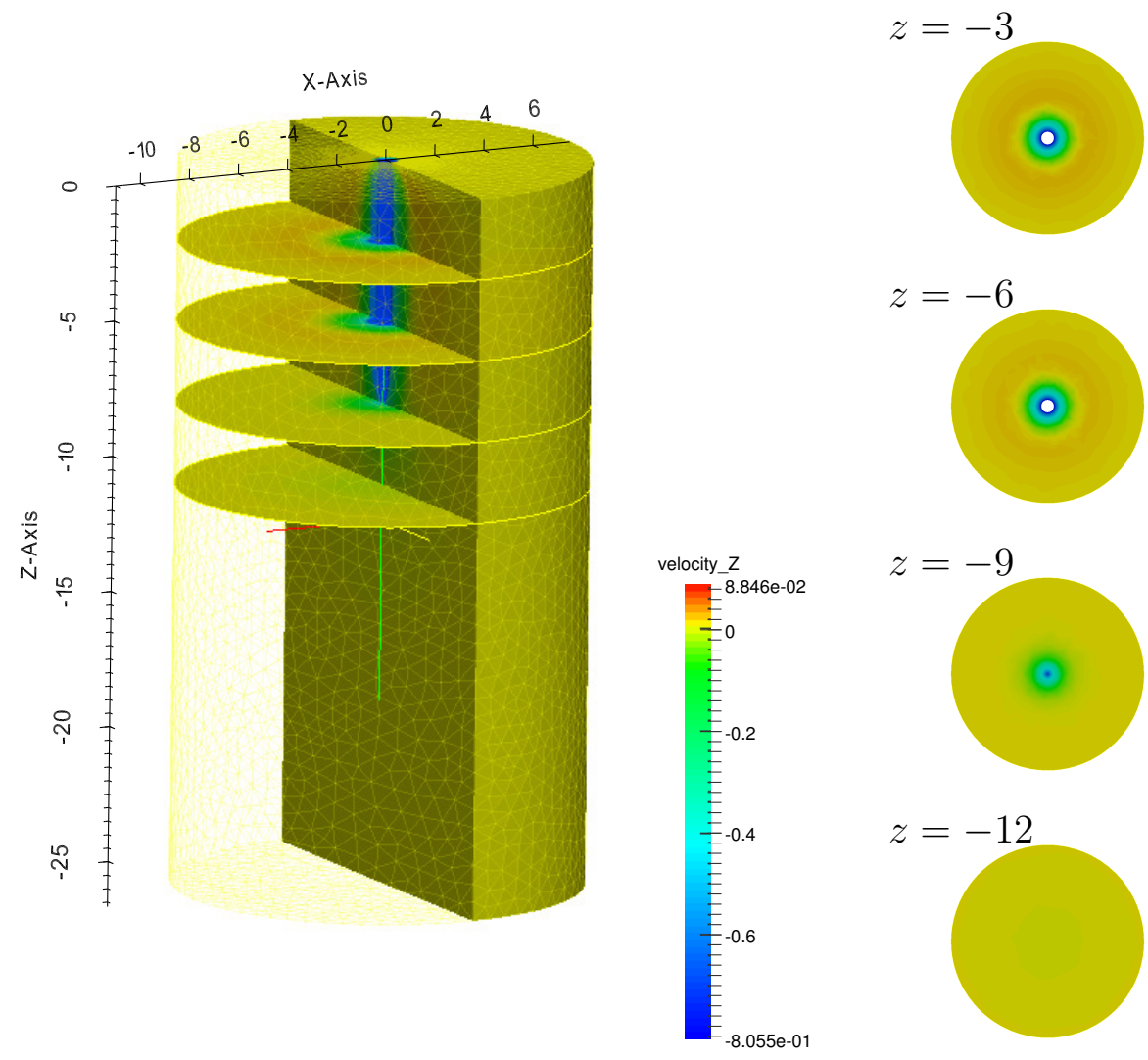

$z=-12$

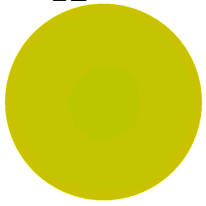

Figure 3: The predicted contours of velocity along the z-direction resulting from the needle (blue) motion in interstitial fluid with $\alpha_{f}=0.7, \mathrm{Da}=0.321$, and $\mathrm{Re}=0.103$

the whole cell surface could be stimulated. Mastocytes have been shown to respond to fluid shear stress [41]. These local mechanical forces participate in the activation of mechanoresponsive proteins on the cell surface [20] so that $\mathrm{Ca}^{++}$is allowed to enter the cytosol via pressure and shear stress gated ion channels. 


\section{Conclusions}

The proposed three-dimensional ALE finite element model is able to describe the interstitial flow and pressure from the macroscopic point of view when a needle is inserted and moved within the hypodermis. High local fluid pressure and shear stress on cells are most likely to appear near the needle tip region. However, the proposed method does not model the deformation of the extracellular matrix and only the effect of interstitial flow is considered. When considering the rotation of the needle, a large deformation of tissues is observed with the twisting of the fibers around the needle, that in turn makes the corresponding change in interstitial flow. A fluid/structure model taking into account the mechanics of the fibers should then be considered. This study has shown that the numerical prediction of the interstitial pres-

o sure and shear stress is an essential tool to gain a better understanding of the activity involved in acupuncture needling.

\section{References}

[1] C. S. Peskin, Numerical analysis of blood flow in the heart, Journal of Computational Physics 25 (3) (1977) 220-252.

[2] R. Glowinski, T.-W. Pan, J. Periaux, A fictitious domain method for dirichlet problem and applications, Computer Methods in Applied Mechanics and Engineering 111 (3-4) (1994) 283-303.

[3] J. Janela, A. Lefebvre, B. Maury, A penalty method for the simulation of fluid-rigid body interaction, in: ESAIM: Proceedings, Vol. 14, EDP Sciences, 2005, pp. 115-123. 
[4] A. Lefebvre, Fluid-particle simulations with FreeFem++, in: ESAIM: Proceedings, Vol. 18, EDP Sciences, 2007, pp. 120-132.

[5] K. Miller, Moving finite elements. II, SIAM Journal on Numerical Analysis 18 (6) (1981) 1033-1057.

225 
stabilization parameters, International Journal for Numerical Methods in Fluids 43 (5) (2003) 555-575.

[12] K. Takizawa, T. E. Tezduyar, Multiscale space-time fluid-structure interaction techniques, Computational Mechanics 48 (3) (2011) 247-267.

[13] C. W. Hirt, A. A. Amsden, J. L. Cook, An arbitrary LagrangianEulerian computing method for all flow speeds, Journal of Computational Physics 14 (3) (1974) 227-253.

[14] T. J. R. Hughes, W. K. Liu, T. K. Zimmermann, Lagrangian-Eulerian finite element formulation for incompressible viscous flows, Computer Methods in Applied Mechanics and Engineering 29 (3) (1981) 329-349.

[15] J. Donea, S. Giuliani, J. P. Halleux, An arbitrary Lagrangian-Eulerian finite element method for transient dynamic fluid-structure interactions, Computer Methods in Applied Mechanics and Engineering 33 (1-3) (1982) 689-723.

[16] C. Grandmont, Y. Maday, Fluid-structure interaction: a theoretical point of view, Revue Européenne des Éléments 9 (6-7) (2000) 633-653.

[17] A. Quarteroni, M. Tuveri, A. Veneziani, Computational vascular fluid dynamics: problems, models and methods, Computing and Visualization in Science 2 (4) (2000) 163-197.

[18] A. Decoene, B. Maury, Moving meshes with FreeFem++, Journal of Numerical Mathematics 20 (2013) 195. 
[19] Y. Deleuze, M. Thiriet, T. W. H. Sheu, Modeling and simulation of local physical stress on the mastocytes created by the needle manipulation during acupuncture, Communications in Computational Physics.

[20] M. Thiriet, Intracellular Signaling Mediators in the Circulatory and Ventilatory Systems, Vol. 4 of Biomathematical and Biomechanical Modeling of the Circulatory and Ventilatory Systems, Springer New York, New York, NY, 2013.

[21] M. Thiriet, Y. Deleuze, T. W. Sheu, A biological model of acupuncture and its derived mathematical modeling and simulations, Communications in Computational Physics.

[22] M. A. Swartz, M. E. Fleury, Interstitial flow and its effects in soft tissues, Annual Review of Biomedical Engineering 9 (1) (2007) 229-256.

[23] J. Y. Park, S. J. Yoo, L. Patel, S. H. Lee, S.-H. Lee, Cell morphological response to low shear stress in a two-dimensional culture microsystem with magnitudes comparable to interstitial shear stress, Biorheology 47 (3) (2010) 165-178.

[24] Y.-H. Tseng, H. Huang, An immersed boundary method for endocytosis, Journal of Computational Physics 273 (2014) 143-159.

[25] M. Thiriet, Cell and Tissue Organization in the Circulatory and Ventilatory Systems, Vol. 1 of Biomathematical and Biomechanical Modeling of the Circulatory and Ventilatory Systems, Springer New York, New York, NY, 2011. 
[26] A. Blasselle, Modélisation mathématique de la peau, Thèse de doctorat, Université Pierre et Marie Curie, Paris, France (2011).

[27] W. Yao, G. H. Ding, Interstitial fluid flow: simulation of mechanical environment of cells in the interosseous membrane, Acta Mechanica Sinica 27 (4) (2011) 602-610.

[28] W. Yao, Y. Li, G. Ding, Interstitial fluid flow: the mechanical environment of cells and foundation of meridians, Evidence-Based Complementary and Alternative Medicine 2012 (2012) 1-9.

[29] M. Thiriet, Biology and Mechanics of Blood Flows: Part I: Biology, CRM Series in Mathematical Physics, Springer, NY, 2008.

[30] M. Thiriet, Cells and tissues, in: Cell and Tissue Organization in the Circulatory and Ventilatory Systems, no. 1 in Biomathematical and Biomechanical Modeling of the Circulatory and Ventilatory Systems, Springer New York, 2011, pp. 11-67.

[31] C. T. Hsu, P. Cheng, Thermal dispersion in a porous medium, International Journal of Heat and Mass Transfer 33 (8) (1990) 1587-1597.

[32] P. Nithiarasu, K. N. Seetharamu, T. Sundararajan, Natural convective heat transfer in a fluid saturated variable porosity medium, International Journal of Heat and Mass Transfer 40 (16) (1997) 3955-3967.

305 [33] K. Vafai, C. L. Tien, Boundary and inertia effects on flow and heat transfer in porous media, International Journal of Heat and Mass Transfer 24 (2) (1981) 195-203. 
[34] J. A. Pedersen, F. Boschetti, M. A. Swartz, Effects of extracellular fiber architecture on cell membrane shear stress in a 3d fibrous matrix, Journal of Biomechanics 40 (7) (2007) 1484-1492.

[35] F. Hecht, New development in FreeFem++, Journal of Numerical Mathematics 20 (3-4) (2013) 251.

[36] M. A. Fernández, L. Formaggia, J.-F. Gerbeau, A. Quarteroni, The Derivation of the Equations for Fluids and Structure, in: L. Formaggia, A. Quarteroni, A. Veneziani (Eds.), Cardiovascular Mathematics, no. 1 in MS\&A, Springer Milan, 2009, pp. 77-121.

[37] A. J. Chorin, A numerical method for solving incompressible viscous flow problems, Journal of Computational Physics 2 (1) (1967) 12-26.

[38] R. Témam, Une méthode d'approximation de la solution des équations de Navier-Stokes, Bulletin de la Société Mathématique de France 96 (1968) 115-152.

[39] P.-A. Raviart, J.-M. Thomas, Introduction À L'analyse Numérique Des Équations Aux Dérivées Partielles, Masson, 1983.

[40] H. Dütsch, F. Durst, S. Becker, H. Lienhart, Low-Reynoldsnumber flow around an oscillating circular cylinder at low Keulegan\&\#8211;Carpenter numbers, Journal of Fluid Mechanics 360 (1998) 249-271.

[41] F. Wei, X. Shi, J. Chen, L. Zhou, Fluid shear stress-induced cytosolic calcium signalling and degranulation dynamics in mast cells, Cell Biology International Reports 19 (2) (2012) 45-51. 

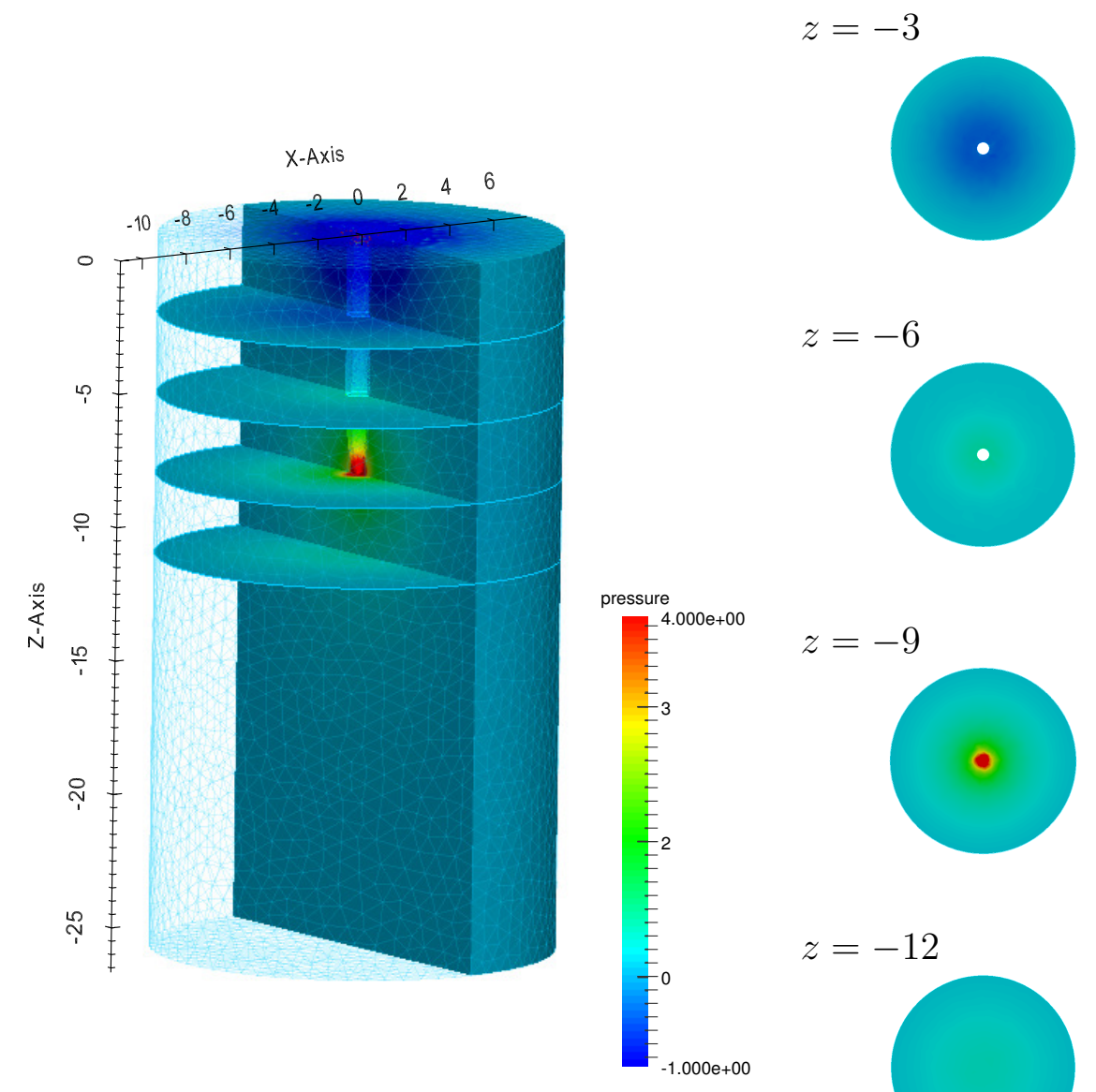

$z=-9$

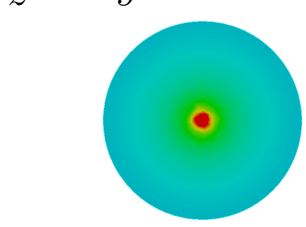

$z=-12$

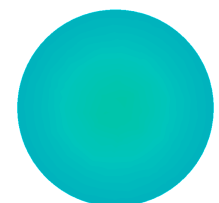

Figure 4: The predicted contours of velocity along the z-direction resulting from the needle (blue) motion in interstitial fluid with $\alpha_{f}=0.7, \mathrm{Da}=0.321$, and $\mathrm{Re}=0.103$. 


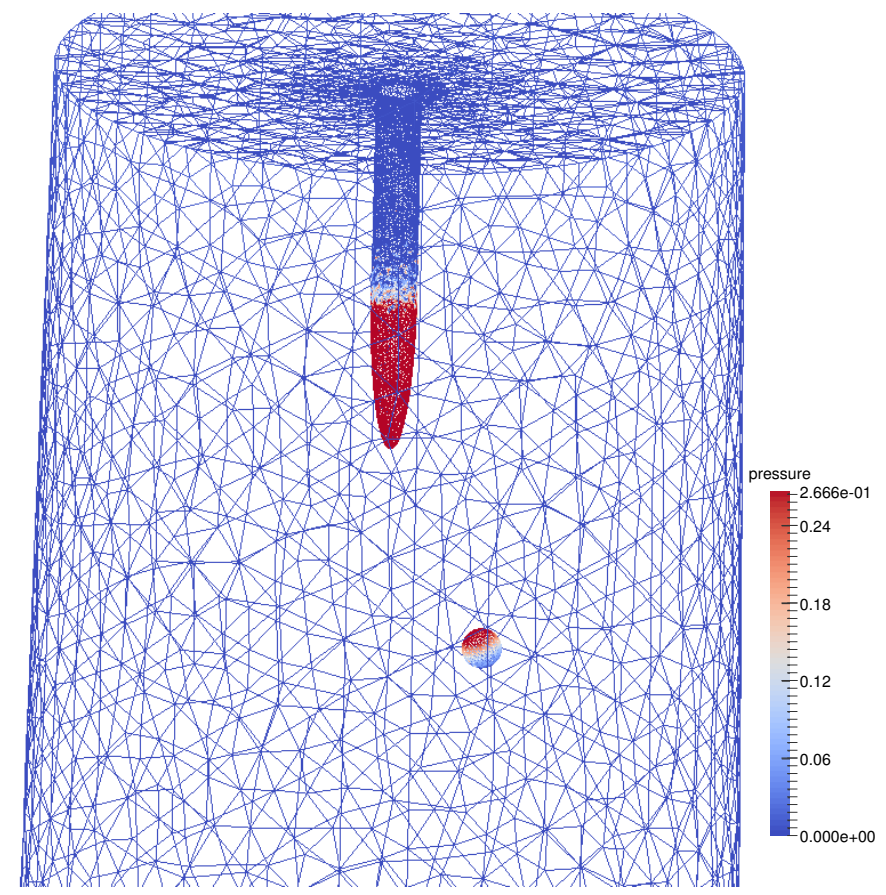

Figure 5: The predicted pressure contours on the needle and cell surface as the needle moves toward the cell with $\alpha_{f}=0.7, \mathrm{Da}=0.321$, and $\mathrm{Re}=0.103$. 


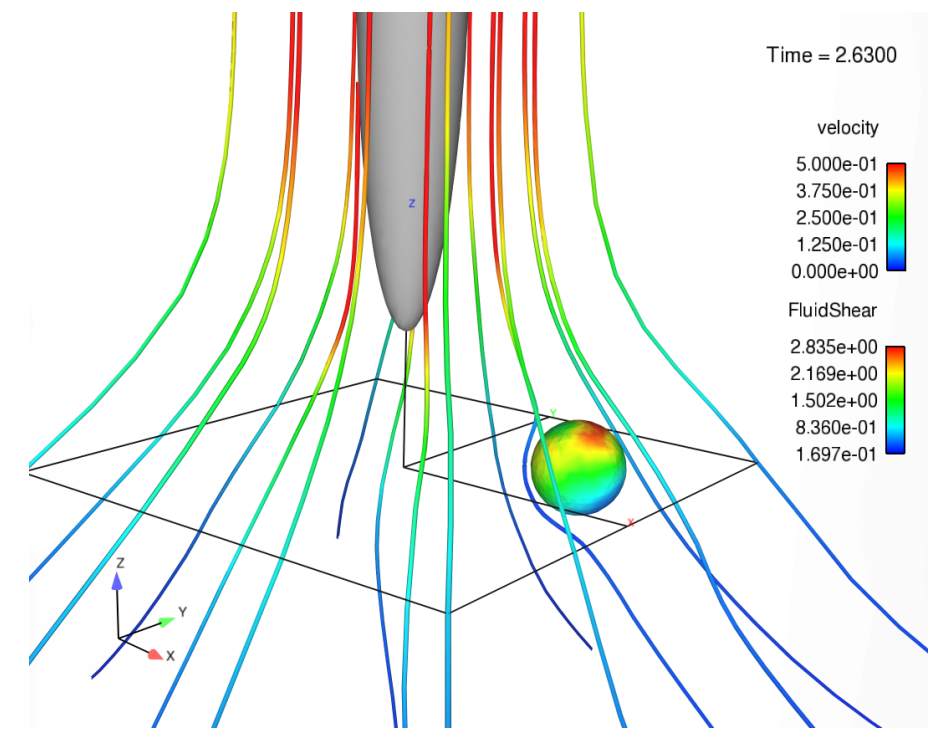

Figure 6: The predicted streamlines and shear stress on the cell surface as the needle moves toward the cell with $\alpha_{f}=0.7, \mathrm{Da}=0.321$, andRe $=0.103$. 